\title{
Modeling the Kinetics, Dynamic Profiles and Strategies for Optimal Efficacy of Photo-Polymerization in Thick Polymers
}

Jui-Teng Lin ${ }^{1}$, Kuo-Ti Chen ${ }^{2}$, Hsai-Wei Liu ${ }^{3}$ and Da-Chuan Cheng ${ }^{4, *}$

1 New Vision Inc., 5F, No. 27, Lane 10, Jiuquan St., Taipei 103, Taiwan;

E-Mail: jtlin55@gmail.com

2 Graduate Institute of Applied Science and Engineering, Fu Jen Catholic University, New Taipei City, Taiwan.

E-mail: tony022199@msn.com

3 Department of Life Science, Fu Jen Catholic University, New Taipei City, Taiwan; E-Mail:079336@gmail.com

4 Department of Biomedical Imaging and Radiological Science, China Medical University, Taichung 404, Taiwan

E-mail: dccheng@mail.cmu.edu.tw

* Author to whom correspondence should be addressed; E-Mail: dccheng@mail.cmu.edu.tw Tel.: +886-4-2205-3366 (ext. 7803); Fax: +886-4-2208-4179. dccheng@mail.cmu.edu.tw

ABSTRACT: The kinetics and optimal efficacy conditions of photoinitiated polymerization are theoretically presented. Analytic formulas are derived for the crosslink time, crosslink depth and efficacy function. The roles of photosensitizer (PS) concentration, diffusion depth and light intensity on the polymerization spatial and temporal profiles, for both uniform and non-uniform cases, are presented. For optimal efficacy, a strategy via controlled PS concentration is proposed, where re-supply of PS in high light intensity may achieve a combined-efficacy similar to low light intensity, but has a much faster procedure. A new criterion of efficacy based on the polymerization (crosslink) [strength] and [depth] is introduced.

Keywords: polymerization modeling; photo polymerization kinetic; photoinitiation rate; polymerization efficacy; crosslinking. 


\section{Introduction}

Photoinitiated polymerization provides advantageous means over the thermalinitiated polymerization, including fast and controllable reaction rates, spatial and temporal control over the formation of the material, and without a need for high temperatures or $\mathrm{pH}$ conditions [1,2]. It also offers controllable process which is light wavelength selective and maximum efficacy can be achieved by optimal photosensitizer (PS) and light parameters such as PS concentration distribution and the control of light path. Commercial type-I photoinitiators with two radicals following photon absorption using visible light absorbance have limited water solubility and high cell toxicity $[3,4]$. Therefore, UV light at $365 \mathrm{~nm}$ has been used for improved polymerization kinetics, at lower initiator concentrations [4]. Photopolymerization for the encapsulation of living cells has proven a useful tool for the study of threedimension cellular behavior for various biomedical applications [1,2].

The kinetics of photoinitiated polymerization systems (PPS) have been studied by many researchers for uniform photoinitiator distribution or for the over simplified cases that the photolysis product becomes completely transparent after polymerization or constant light intensity [4-6]. For more realistic systems, the distribution of the photoinitiator is non-uniform and the UV light may still be absorbed by the photolysis product besides the absorption of the monomer. Therefore, the kinetics of PPS becomes very complex and the process is governed by multiple factors. To improve the efficiency of PPS particularly in a thick system $(>1.0 \mathrm{~cm})$, we have presented the numerical results using a focused light [7] and two-beam approach for the case of uniform PS distribution [8] and analytic comprehensive modeling for the non-uniform case [9].

The kinetics of PPS may be described by type-I photoinitiators dissociate into two radicals following photon absorption; or type-II initiating systems, in which the excited state after photon absorption, abstracts a hydrogen atom from a second, coinitiator species [1,2]. However, type II photoinitiator requires a coinitiator and often accelerant species to generate efficiently a sufficient number of radicals for photopolymerization. The kinetic of PPS and its clinical applications for anti-cancer [10-13] and corneal crosslinking (CXL) $[14,15]$ have been recently presented. We have presented modeling the efficacy profiles of UV-light activated CXL, in which the treated cornea has a much smaller thickness (approximately $500 \mathrm{um}$, or $0.05 \mathrm{~cm}$ ) than most polymer systems, approximately $1.0 \mathrm{~cm}$. Therefore, the PPS depth-profile and optimal features in thick polymers require further studies. Accelerated CXL has been clinically used for faster procedure (within 3 to 10 minutes) using higher light intensity of 9 to $45 \mathrm{~mW} / \mathrm{cm}^{2}$, in replacing the conventional CXL of $3 \mathrm{~mW} / \mathrm{cm}^{2}$ which 
took 30 minutes [14]. However, no efforts have been done for fast PPS in thick polymers using high light intensity.

For practical and/or clinical purpose, the preferred parameters of PPS include: minimum dose (or fluence), fast procedure, minimum cell toxicity, minimum concentration, maximum and uniform reactive depth, and maximum efficacy. However, certain of these parameters are competing factors, and therefore optimal condition is required for best outcomes. Furthermore, environment conditions such as the internal and external amount of oxygen and PS concentration control are critical in determining the efficacy.

In this study, we will present detail analysis for the roles of each of the key parameters including the PS initial concentration and its distribution, the light intensity and the kinetic rate constants. The important feature of optimal photoinitiation rate is explored by the balance of the two competing factors, the light intensity and the initiator concentration. we will investigate the roles of PS initial concentration and light intensity and fluence on the spatial and temporal profiles of the efficacy function, for both uniform and non-uniform cases. For optimal efficacy, strategy via controlled PS concentration will be presented, for the first time, where re-supply of PS concentration during the PPS is defined by a polymerization (crosslink) time which is inverse proportional to the light intensity [15]. Furthermore, we will define a new criterion of efficacy based on the polymerization (crosslink) strength and depth.

\section{Methods and Modeling systems}

\subsection{Photochemical kinetics}

As shown in Fig. 1, the photochemical kinetics has three pathways $[13,16]$. Defining $\left[\mathrm{S}_{0}\right],\left[\mathrm{S}_{1}\right]$ and $\left[\mathrm{T}_{3}\right]$ are the ground state, singlet excited state, and triplet excited state of PS molecules which is excited by the UV light. In type-I pathway, $\mathrm{T}_{3}$ can interact directly with the collagen substrate (A); or with the ground state oxygen $\left(\mathrm{O}_{2}\right)$ to generate a superoxide anion $\left(\mathrm{O}^{-}\right)$; in type-II pathway, $\mathrm{T}_{3}$ interacts with the ground oxygen $\left(\mathrm{O}_{2}\right)$ to form a reactive singlet oxygen $\left(\mathrm{O}^{*}\right)$ [16]. The PS excited triplet can undergo two kinds of reactions. In type I reaction, it can react directly with a substrate (cell membrane or a molecule), and transfer a proton or an electron to form a radical anion or radical action, respectively. These radicals may further react with oxygen to produce reactive oxygen species (ROS). Alternatively, in a type II reaction, the triplet RF can transfer its energy directly to molecular oxygen (triplet in the ground state), to form excited- state singlet oxygen. As show by Fig. 1, both type-I and type-II reactions can occur simultaneously, and the ratio between these processes depends on the types and the concentrations of PS, substrate and oxygen, the kinetic 
rates involved in the process. For example, in rose Bengal, type-II is the predominant process with singlet oxygen contributing about $80 \%$; and for riboflavin, they are $49 \%$ and $1 \%$, respectively [16].

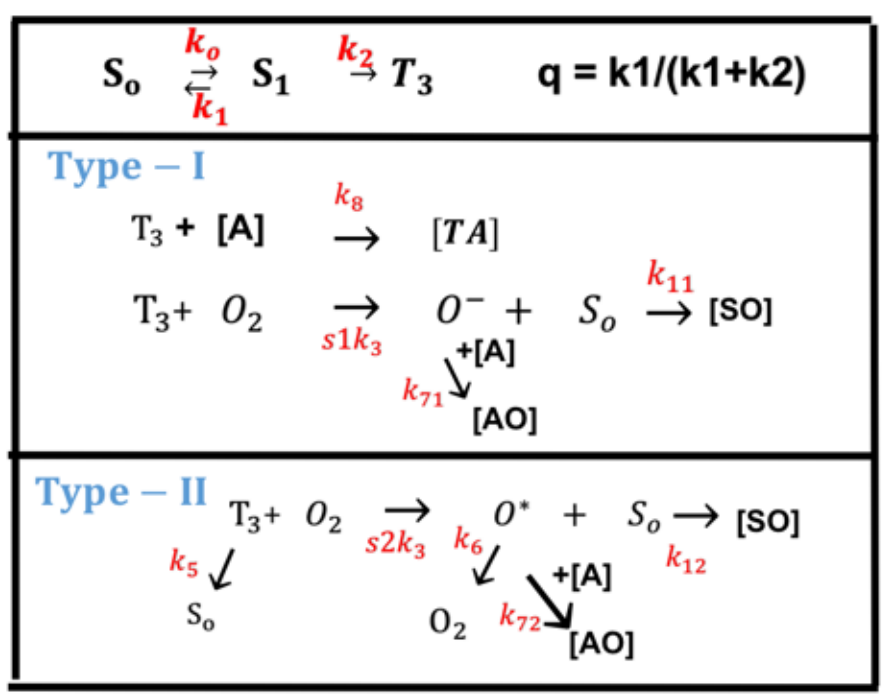

Fig. 1. The photochemical kinetics, where $\left[\mathrm{S}_{0}\right],\left[\mathrm{S}_{1}\right]$ and $\left[\mathrm{T}_{3}\right]$ are the ground state, singlet excited state, and triplet excited state of PS molecules (see text for more details)[16].

These factors also influence the overall photopolymerization efficacy, particularly the PS triplet state quantum yield (q) and its concentration. Furthermore, the specific protocols and the methods of PS instillations prior to and during the photopolymerization also affect the short and long term outcomes. The overall photopolymerization efficacy basically is proportional to the time integration of the UV light intensity, I $(z, t)$ and the PS and oxygen concentration, $\mathrm{C}(\mathrm{z}, \mathrm{t})$, and $\left[{ }^{3} \mathrm{O}_{2}\right]$. The efficacy reaches a saturated (steady) state when $\mathrm{C}(\mathrm{z}, \mathrm{t})$ or $\left[{ }^{3} \mathrm{O}_{2}\right]$ is depleted by the light, where higher intensity depletes $\mathrm{C}(\mathrm{z}, \mathrm{t})$ and $\left[{ }^{3} \mathrm{O}_{2}\right]$ faster and therefore reaches a lower steady-state efficacy. For clinical application of CXL, the Dresden (at 3 $\mathrm{mW} / \mathrm{cm}^{2}$ ) protocol, therefore, is always more effective than the accelerated CXL with 9 to $45 \mathrm{~mW} / \mathrm{cm}^{2}$. However, this drawback maybe overcame by a concentrationcontrolled method (CCM) first proposed by Lin [15].

Referring to the three kinetic pathways of Fig. 1, a set of macroscopic kinetic equation for the RF ground-state, $\mathrm{C}(\mathrm{z}, \mathrm{t})$, and the ground state oxygen molecule $\left[\mathrm{O}_{2}\right]$ (at the quasi steady-state) is constructed $[12,13,16]$

$\frac{\partial \mathrm{C}(\mathrm{z}, \mathrm{t})}{\partial \mathrm{t}}=-\mathrm{b}\left[\mathrm{g}+\mathrm{g}^{\prime}\right] \mathrm{C}$ 
$\frac{\partial Y}{\partial t}=-b s N G+P$

$\frac{\partial \mathrm{I}(\mathrm{z}, \mathrm{t})}{\partial \mathrm{z}}=-\mathrm{A}^{\prime}(\mathrm{z}, \mathrm{t}) \mathrm{I}(\mathrm{z}, \mathrm{t})$

$\mathrm{A}^{\prime}(\mathrm{z}, \mathrm{t})=2.3\left[\left(\mathrm{a}^{\prime}-\mathrm{b}^{\prime}\right) \mathrm{C}(\mathrm{z}, \mathrm{t})+\mathrm{b}^{\prime} \mathrm{C}_{0}(\mathrm{z})+\mathrm{Q}\right]$

where $\mathrm{b}=\mathrm{aqI}(\mathrm{z}, \mathrm{t}) ; \mathrm{a}=83.6 \mathrm{wa} \mathrm{a}^{\prime} ; \mathrm{w}$ is the light wavelength; a' and $\mathrm{b}^{\prime}$ are the molar extinction coefficient of the initiator and the photolysis product, respectively; $Q$ is the absorption coefficient of the monomer and the polymer repeat unit. Typical values are [4,9]: $\mathrm{a}^{\prime}=0.2$ to $0.3(1 / \mathrm{mM} / \mathrm{cm}), \mathrm{b}^{\prime}=0.1$ to $\left.0.15(1 / \mathrm{mM} / \mathrm{cm})\right)$, and $\mathrm{C}_{0}=1.0$ to $3.0 \mathrm{mM}$; and for a UV light at $365 \mathrm{~nm}, \mathrm{a}=0.00305 \mathrm{a}^{\prime}(1 / \mathrm{cm})$.

For type-I, g=k $\mathrm{k}_{8}[\mathrm{~A}] \mathrm{G}_{0} / \mathrm{k}_{3}, \mathrm{G}_{0}=/\left(\left[\mathrm{O}_{2}\right]+\mathrm{k}+\mathrm{K}^{\prime}\right)$; and for type-II, g',=K' $\mathrm{G}(\mathrm{z})$, with $\mathrm{G}(\mathrm{z})=(\mathrm{C}+\mathrm{c}) \mathrm{G}_{0}, \mathrm{~K}^{\prime}=\mathrm{k}_{12} /\left(\mathrm{k}_{6}+\mathrm{k}_{12}(\mathrm{C}+\mathrm{c})+\mathrm{k}_{72}[\mathrm{~A}]\right)$; $\mathrm{c}$ is a low concentration correction related to the diffusion of singlet oxygen $[12,13]$. $\mathrm{q}$ is the triplet state [T] quantum yield given by $\mathrm{q}=\mathrm{k}_{2} /\left(\mathrm{k}_{1}+\mathrm{k}_{2}\right) ; \mathrm{s}=\mathrm{s} 1+\mathrm{s} 2$, with $\mathrm{s}_{1}$ and $\mathrm{s}_{2}$ are the fraction of $\left[\mathrm{O}_{2}\right]$ converted to the singlet oxygen and other ROS, respectively, in type-I and type-II [16].

In Eq. (1.b) we have included a fit parameter ( $N=5$ to 10$)$ which can be fit to the measured oxygen profiles at various UV intensity [16]. And the UV light intensity in the polymer is given by a time-dependent Beer-Lambert law [14]. We have also included in Eq. (1.b) the oxygen source term $P(z, t)=p\left(1-X / X_{0}\right)$, with a rate constant $p$ to count for the situation when there is an external continuing supply, or nature replenishment (at a rate of $\mathrm{p}$ ), besides the initial oxygen in the polymer.

We note that Eq. (1) was also presented by Kim et al [12,13] for the anti-cancer kinetics. However, they have assumed a constant UV intensity, i.e., A' $(z, t)$ is a constant in Eq. (1.d). They also ignored the contribution from the type-I term, $\mathrm{k}_{8}$ [A], since typeII is dominant in their anti-cancer process. Most of the previous model have also ignored the dynamic of UV intensity given by Eq. (1.c) and the depth-dependent profile of PS and UV intensity [4-6]. Exact solutions of Eq. (1) require numerical simulations. For analytic formulas [9], we will use an effective $A(z, t)$ or its mean value, such that $\mathrm{A}^{\prime}(\mathrm{z}, \mathrm{t})$ becomes time-independent in solving Eq. (1).

\subsection{Dynamic Concentration Profile}

In solving Eq. (1), we will choose initial profile (at $\mathrm{t}=0) \mathrm{I}_{0}(\mathrm{z})=\mathrm{I}_{0}(1-0.25 \mathrm{z} / \mathrm{D})$ for the light intensity; and $\mathrm{C}_{0}(\mathrm{z})=\mathrm{C}_{0} \mathrm{~F}(\mathrm{z})$, with $\mathrm{F}(\mathrm{z})=1-0.5 \mathrm{z} / \mathrm{D}$, for the PS concentration distribution; where $\mathrm{D}$ is the PS concentration distribution depth; and when $\mathrm{D}>>1.0 \mathrm{~cm}$, $\mathrm{F}=1$ representing a flat (or uniform) PS distribution. Analytic solution of Eq. (1) is available for the type-I process and under certain approximation. For $g>>$ g', or for the case that type-I is dominant over type-II, we obtain an approximate solution, $\mathrm{C}(\mathrm{z}, \mathrm{t})=$ 
$\mathrm{C}_{0} \mathrm{~F}(\mathrm{z}) \exp (-\mathrm{bgt})$, assuming $\mathrm{b}$ and $\mathrm{g}$ are time-independent, or taking their averaged value. $A^{\prime}(z, t)$ in Eq. (1.d) has an initial value (A1, with $\left.b^{\prime}=0\right)$ and steady state value (A2, with $\mathrm{C}=0)$, given by : $\mathrm{A} 1=2.3\left[\mathrm{a}^{\prime} \mathrm{C}_{0} \mathrm{~F}^{\prime}+\mathrm{Q}\right]$, and $\mathrm{A} 2=2.3\left[\mathrm{~b}^{\prime} \mathrm{C}_{0} \mathrm{~F}^{\prime}+\mathrm{Q}\right]$, with $\mathrm{F}^{\prime}(\mathrm{z})=1-0.25 \mathrm{z} / \mathrm{D}$ being the integration of $F(z)$ over $z$; the mean value is given by $A=0.5(A 1+A 2)$. We have also developed numerically fit value [9], $\mathrm{A}=2.3\left[\mathrm{mb}^{\prime} \mathrm{C}_{0} \mathrm{~F}+\mathrm{Q}\right]$, with $\mathrm{m}=0.8$ to 1.0 depending on the value of a' and b'.

A depletion time $\mathrm{T}^{*}$ may be defined by when $\mathrm{C}\left(\mathrm{z}, \mathrm{t}=\mathrm{T}^{*}\right)=\mathrm{C}_{0}(\mathrm{z}) \exp (-\mathrm{M})$, with $\mathrm{M}$ $=2$, or $\mathrm{C}(\mathrm{z}, \mathrm{t})$ is depleted to 0.13 of its initial value. We obtain an analytic formula $T^{*}(z)=T_{0} \exp (A z)$, where $T_{0}$ is the surface depletion time given by $T_{0}=M /(b g)$, which is inverse proportional to the UV light initial intensity, since $b=a q I(z) . T^{*}$ may be also defined by the level of photopolymerization efficacy, or the crosslink time (Tc), to be discussed later. The strong depletion of $\mathrm{C}(\mathrm{z}, \mathrm{t})$ will also affect the time-dependent profiles of the intensity, I $(\mathrm{z}, \mathrm{t})$, which in general, will not follow the conventional Beer-Lambert law (BLL), and should be governed by a generalized, time-dependent BLL first presented by Lin et al [17].

\subsection{Efficacy Profiles}

The normalized photo-polymerization efficacy defined by Ceff $=1-[\mathrm{A}] /[\mathrm{A}]_{0}=1$ $\exp (-\mathrm{S})$, with $\mathrm{S}$-function for type-I $\left(\mathrm{S}_{1}\right)$ and type-II $\left(\mathrm{S}_{2}\right)$, and the overall efficacy is given by Ceff=1-exp[-( $\left.\left.\mathrm{S}_{1}+\mathrm{S}_{2}\right)\right]$. The type-I efficacy may be further expressed by rate equation of conversion of collagen monomers $[\mathrm{M}]$ to polymers, where the NOM term of Eq. (1.a), $\mathrm{g}=\mathrm{k}_{8}[\mathrm{~A}] \mathrm{G}_{0} / \mathrm{k}_{3}$, is replaced by an overall rate constant $(\mathrm{K})$ including all polymerization chain reactions. The $\mathrm{S}$ functions are given by [16]

$\mathrm{S}_{1}=\int_{0}^{\mathrm{t}}\left[\sqrt{\operatorname{aqgK~IC}}+\left(f \mathrm{~s}_{1} \mathrm{aqK}^{\prime}\right) \mathrm{I}(\mathrm{z}, \mathrm{t}) \mathrm{G}\right] \mathrm{dt}$

for type-I, and

$\mathrm{S}_{2}=\int_{0}^{\mathrm{t}} f \mathrm{~s}_{2} a q K^{\prime} \mathrm{I}(\mathrm{z}, \mathrm{t}) \mathrm{G} d \mathrm{t}$

for type-II.

The first term in Eq. (3.a) relates to the direct coupling of triplet state [T] with the substrate [A] under hypoxic conditions or any other non-oxygen-mediated (NOM) reactions; and the second term relates with the (ROS)-mediated reactions (in type-I). $\mathrm{f}$ is the fraction of all ROS (including singlet oxygen) interacting with acceptors $[\mathrm{A}]$, or the oxygen-mediated (NOM) reactions in type-I and type-II. S $\mathrm{s}_{2}$ and $\mathrm{s}_{1}$ are the fraction of $\left[\mathrm{O}_{2}\right]$ interacting with $[\mathrm{T}]$ to produce singlet oxygen (in type-II) and other ROS (in type-I), respectively.

$\mathrm{S}_{2}$ requires numerical integration of Eq. (3.b) which was shown elsewhere for the anti-cancer system with type-II being the dominant process [11]. We will focus on 
the type-I process which is more common in photo-polymerization. For analytic formulas, we will use the mean value of $\mathrm{A}(\mathrm{z})$ such that $\mathrm{I}(\mathrm{z}, \mathrm{t})=\mathrm{I}_{0} \exp (-\mathrm{Az})$, and and $\mathrm{C}(\mathrm{z}, \mathrm{t})=\mathrm{C}_{0} \mathrm{Fexp}(-\mathrm{Bt})$, with $\mathrm{B}=\mathrm{bg}=\operatorname{aqgI}(\mathrm{z})$, Eq. (3.a), for the case that $\mathrm{g}^{\prime}<<\mathrm{g}$, the type-I $\mathrm{S}$ function is given by [16]

$$
\begin{aligned}
& S_{1}=\sqrt{4 K C o F \exp (A z) /(a q g I o)} E 1 \\
& E 1=[1-\exp (-0.5 B t)]
\end{aligned}
$$

which is a nonlinear function of E given by its Taylor expansion $S=$ $\sqrt{(a q I o K C o F) \exp (-A z)} t[1-0.5 a q g E+\cdots]$, which follows BRL only for small time with the first term kept. In contrast, type-II efficacy, given by the time integral of [IC] follows the BRL [16]. A crosslink time (Tc) may be defined by Eq. (4.b) when $\mathrm{E} 1=0.87$, or $0.5 \mathrm{BTc}=2$, which gives us $\mathrm{T} c=4 / \mathrm{B}=4 /(\mathrm{bg})=[4 /(\operatorname{aqgI})] \exp (\mathrm{Az})=\mathrm{T}_{0}$ $\exp (\mathrm{Az})$, with the surface crosslink time given by $\mathrm{T}_{0}=300 / \mathrm{I}_{0}$, for aqg $=0.01333$. We note that this crosslink time equals to the depletion time $\left(\mathrm{T}^{*}\right)$, when $\mathrm{M}=4$.

\subsection{Optimal Efficacy}

$\mathrm{S}_{1}$ has maximum value at a crosslink depth $\left(\mathrm{z}=\mathrm{z}^{*}\right)$ given by taking $\mathrm{d} \mathrm{S}_{1} / \mathrm{dz}=0$. For the case of $\mathrm{F}=1.0$ (or uniform PS concentration, with $\mathrm{D}>>1.0 \mathrm{~cm}$ ), it is given by the condition of $\mathrm{Bt}=1.25$, which gives an analytic formula $\mathrm{z}^{*}=(1 / \mathrm{A}) \ln \left(\mathrm{E}^{\prime} / 1.25\right)$, with $\mathrm{E}^{\prime}=\mathrm{aqgE}_{0}$. And the corresponding PS concentration is given by $\mathrm{C}_{0}{ }^{*}=\left[\ln \left(\mathrm{E}^{\prime} / 1.25\right)\right] / \mathrm{z}-$ $2.3 \mathrm{Q}] /\left[1.13\left(\mathrm{a}^{\prime}+\mathrm{b}^{\prime}\right) \mathrm{F}\right]$; the maximum $\mathrm{S} 1$ is given by when $\mathrm{E} 1=0.714$, and $\mathrm{z}=\mathrm{z}^{*}$ in $\mathrm{Eq}$. (4). For the case of non-uniform PS concentration (with D is 0.5 to $1.0 \mathrm{~cm}$ ), these optimal conditions require numerical calculations to find the peaks of $\mathrm{S} 1$. We note for $\mathrm{Z}^{*}<1.5 \mathrm{~cm}$, we need large value of $\mathrm{A}$ (or $\mathrm{C}_{0}$ ) and a minimum dose $\left(\mathrm{E}_{0}\right)$, such that $\ln \left(E^{\prime} / 1.25\right)>0$. Furthermore, for a given $A, z^{*}$, which defines the depth of crosslink is proportional to the light dose.

\subsection{Maximum intensity}

High light intensity may be used to reduce the crosslink time. However, there is a minimal time, or maximum intensity $\mathrm{I}^{*}=\mathrm{E}_{0} / \mathrm{t}$, to achieve a threshold efficacy $(\mathrm{St})$. such that $\mathrm{S} 1$ (at $\mathrm{z}=0$, and $\mathrm{Bt}>>1)>\mathrm{St}$. Choosing $\mathrm{St}=2.0$, or efficacy $\mathrm{Eff}=1-\exp (-$ $\mathrm{St})=0.87$, that is $87 \%$ of the polymers are converted to monomers. From eq. (4), we obtain the maximum (or cutoff) intensity $\mathrm{I}^{*}=\mathrm{RC}_{0}$, with $\mathrm{R}=\mathrm{K} /(\mathrm{aqg})$, and the associate minimum irradiation time is $\mathrm{t}^{*}=\mathrm{E}_{0} / \mathrm{I}^{*}$. For example, for $\mathrm{C}_{0}=2.0(1 / \mathrm{mM} / \mathrm{cm})$ and a threshold dose (fluence) $\mathrm{E}_{0}=3.0 \mathrm{~J} / \mathrm{cm}^{2}$, we obtain $\mathrm{I}^{*}=(10,50,100) \mathrm{mW} / \mathrm{cm}^{2}$, for $\mathrm{R}=$ $(5,50,100)$; and $t^{*}=(200,40,20) \mathrm{s}$. This maximum (cutoff) intensity limits the validation of BRL for accelerated PPS, and the minimum irradiation time required for efficient crosslink. This feature was measured clinically in CXL [16], but not yet in 
PPS.

2.6 Nonlinear scaling law

As predicted by our S1 formula, the efficacy at transient state (for small dose) is proportional to $\mathrm{tI}_{0}{ }^{0.5}$, however, at steady-state, it is a nonlinear increasing function of $\left[\mathrm{C}_{0} / \mathrm{I}_{0}\right]^{0.5}$ or $\left[\mathrm{t} / \mathrm{E}_{0}\right]^{0.5}$. This nonlinear scaling law predicts the clinical data more accurate than the linear theory of Bunsen Roscoe law (BRL) [16]. Accelerated PPS based on BRL, therefore, has undervalued the exposure time ( $\mathrm{t}$ ) for higher intensity using the linear scaling of $\mathrm{t}=\left[\mathrm{E}_{0} / \mathrm{I}_{0}\right]$, rather than $\mathrm{t}=\left[\mathrm{E}_{0} / \mathrm{I}_{0}\right]^{0.5}$, based on our nonlinear law. To achieve the same efficacy, higher PS concentration requires higher UV light intensity; and for the same dose, higher UV light intensity requires a longer exposure time.

The BRL is based on the conventional Beer-Lambert law for UV light intensity without PS depletion, such that $\mathrm{I}(\mathrm{z})$ is time-independent, and $\mathrm{C}(\mathrm{z}, \mathrm{t})=\mathrm{constant}=\mathrm{C}_{0} \mathrm{~F}$, therefore, $S_{1}=\sqrt{4 K C o F E o \exp (A z)}$ which is a linear function of the dose $\mathrm{E}_{0}=$ (tI).

Our nonlinear law, as shown by Eq. (4) predicts that high UV intensity requires longer exposure time than what is calculated based on BRL. In addition, for the same dose, higher intensity depletes the PS faster and reach a lower steady-state efficacy. Further discussion will be shown later. As shown by our S-formula, diffusion depth (D) also pays important role. Larger D will achieve higher efficacy as shown by the PS distribution function, $F(z)=1-0.5 z / D$, which is an increasing function of $D$, and $\mathrm{F}=1.0$ for the flat (uniform) distribution case. The above features have been clinically shown in CXL [16], but not yet for PPS.

\section{Results and Discussions}

\subsection{Concentration profiles}

Numerical results of Eq. (1) are shown in Fig. 1 for the PS concentration dynamic profiles. One may see that depletion of PS starts from the polymer surface, and gradually into the volume $(\mathrm{z}>0)$. Fig. 2 shows the normalized depletion time $\left(\mathrm{T}^{*} / \mathrm{T}_{0}\right)$ versus PS concentration, based on analytic formula, $T^{*}(z)=T_{0} \exp (A z)$, where uniform distribution has a longer depletion time than that of non-uniform having a smaller A'.
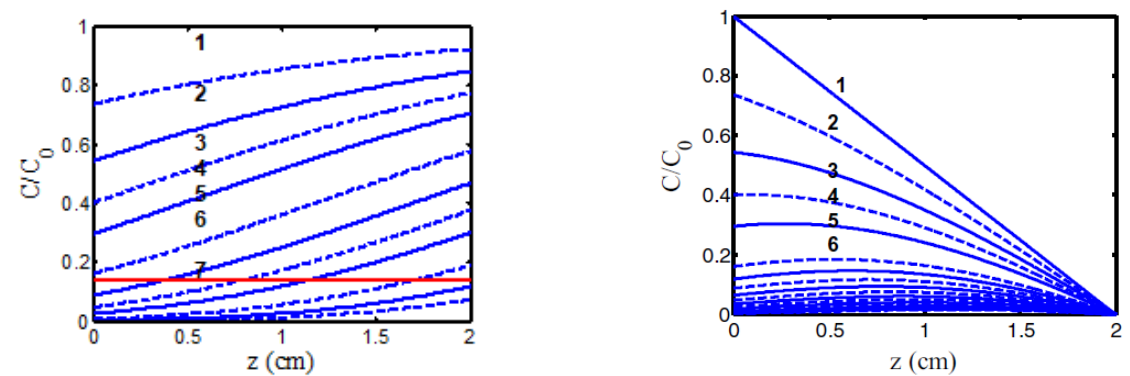
Fig.1 The normalized photoinitiator concentration profiles [9] for uniform (left figure) and non-uniform (right figure, with $\mathrm{D}=1.0 \mathrm{~cm}$ ) $\mathrm{PS}$ distribution, for $\mathrm{t}=0$ (curves 1 ) and $\mathrm{t}=50,100,150,200,300,350$ seconds (curves 2 to 7 ) with quantum yield $\mathrm{q}=1.0$, $\mathrm{a}^{\prime}=0.2(\mathrm{mM} \cdot \mathrm{cm})^{-1}, b^{\prime}=0.15(\mathrm{mM} \cdot \mathrm{cm})^{-1}$, and $\mathrm{Q}=0.1(1 / \mathrm{cm})$.
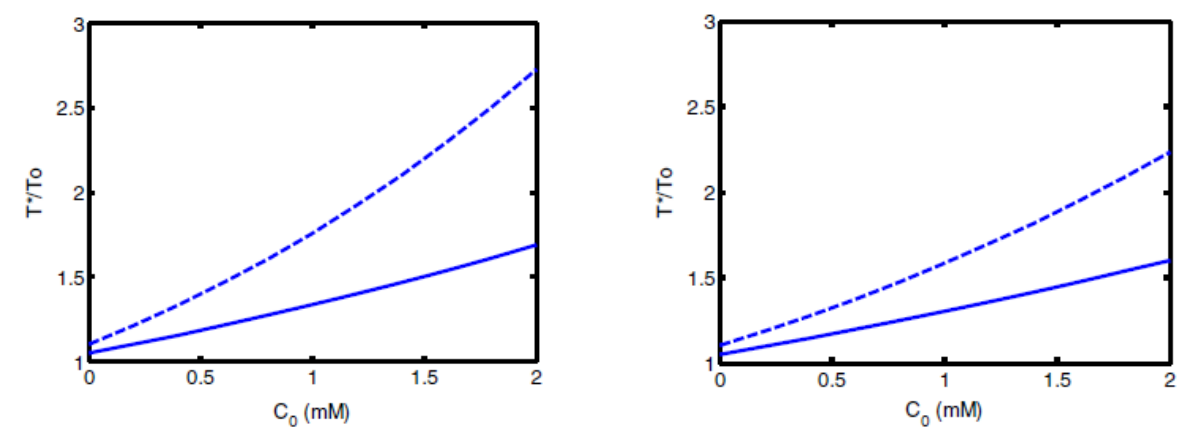

Fig. 2. The normalized depletion time $\left(\mathrm{T}^{*} / \mathrm{T}_{0}\right)$ versus PS initial concentration $\mathrm{C}_{0}$, for uniform (left figure) and non-uniform (right figure), at $\mathrm{z}=0.5$ (solid curve) and $1.0 \mathrm{~cm}$ (dashed curve) [9].

\subsection{Efficacy profiles}

We choose typical values of: $\mathrm{a}^{\prime}=0.2(1 / \mathrm{mM} / \mathrm{cm}), b^{\prime}=0.1(1 / \mathrm{mM} / \mathrm{cm}), Q=0.1(1 / \mathrm{cm})$, $\mathrm{q}=0.5$, aqg $=0.012 \mathrm{~cm}^{2} / \mathrm{mW}$; the mean $\mathrm{A}(\mathrm{z})=0.35 \mathrm{C}_{0} \mathrm{~F}(\mathrm{z})+0.23, \mathrm{~B}=\left(0.006 \mathrm{I}_{0}\right) \exp (-\mathrm{Az})$, with $\mathrm{C}_{0}$ in $\mathrm{mM}, \mathrm{I}_{0}$ in $\mathrm{mW} / \mathrm{cm}^{2}$. Eq. (4) gives a normalized $\mathrm{S}$-function defined by $\mathrm{S}=\mathrm{S}_{1} / \mathrm{S}_{0}$, where $\mathrm{S}_{0}=[4 \mathrm{~K} /(\mathrm{aqg})]^{0.5}$ is a proportional constant, such that

$S=E 1 \sqrt{(\operatorname{CoF} / I o) \exp (A z)}$

Based on Eq. (5), we will investigate the roles of $\mathrm{C}_{0}, \mathrm{I}_{0}$ and $\mathrm{D}$ on the spatial (z) and temporal $(\mathrm{t})$ profiles of $\mathrm{S} 1$, for both uniform and non-uniform cases. In the follow figures, we show the normalized $\mathrm{S}$-function based on Eq. (5) for $\mathrm{S}_{0}=4$, or $\mathrm{K} /(\mathrm{aqg})=4$. In addition, the transient factor $\mathrm{E} 1$ is based on $\mathrm{aqg}=0.012$, or $\mathrm{B}=(\mathrm{aqg}) \mathrm{I}(\mathrm{z})=0.012 \mathrm{I}(\mathrm{z})$, and $\mathrm{K}=4$ (aqg) $=0.048$.

Fig. 4 shows the time profiles of $\mathrm{S}$ versus time (left figure) and versus dose (right figure) for various light intensity $\mathrm{I}_{0}=(10,15,20,30) \mathrm{mW} / \mathrm{cm}^{2}$, for $\mathrm{D}=1.0 \mathrm{~cm}, \mathrm{C}_{0}=3 \mathrm{mM}$ , at $\mathrm{z}=0.5 \mathrm{~cm}$. Fig. 5 shows the spatial profiles of $\mathrm{S}$ for various exposure time, $\mathrm{t}=$ $(100,200,300,400) \mathrm{s}$. Fig. 6 compares profiles for low and high light intensity at $\mathrm{I}_{0}=10$ (left figure) and 20 (right figure) $\mathrm{mW} / \mathrm{cm}^{2}$, for the non-uniform case with $\mathrm{D}=1.0 \mathrm{~cm}$. Fig. 7 shows the role of $\mathrm{D}$, with $\mathrm{D}=0.5 \mathrm{~cm}$ (with a cutoff at $2 \mathrm{D}=1.0 \mathrm{~cm}$ ), and $\mathrm{D}=20 \mathrm{~cm}$ (uniform case). 
Fig. 8 shows $\mathrm{S}$ versus PS concentration $\left(\mathrm{C}_{0}\right)$, for $\mathrm{z}=0$ and $0.5 \mathrm{~cm}$, for $\mathrm{D}=1.0 \mathrm{~cm}$, $\mathrm{I}_{0}=10 \mathrm{~mW} / \mathrm{cm}^{2}$. Fig. 9 shows $\mathrm{S}$ versus light intensity $\left(\mathrm{I}_{0}\right)$, for for $\mathrm{z}=0$ and $0.5 \mathrm{~cm}$, for $\mathrm{D}=1.0 \mathrm{~cm}, \mathrm{C}_{0}=2 \mathrm{mM}$, for $\mathrm{t}=100 \mathrm{~s}$ (red curve) and $200 \mathrm{~s}$ (green curve).

We note that the time of transient factor E1 is scaled by (aqg) and the $\mathrm{S}_{1}$ function is scaled by $\mathrm{S}_{0}=[4 \mathrm{~K} /(\mathrm{aqg})]^{0.5}$. Therefore, the above profiles maybe easily re-produced for a given values of $\mathrm{K}$ and aqg, when different PS is used having different absorption coefficient (a), quantum yield (q) or effective kinetic rate constant (K).
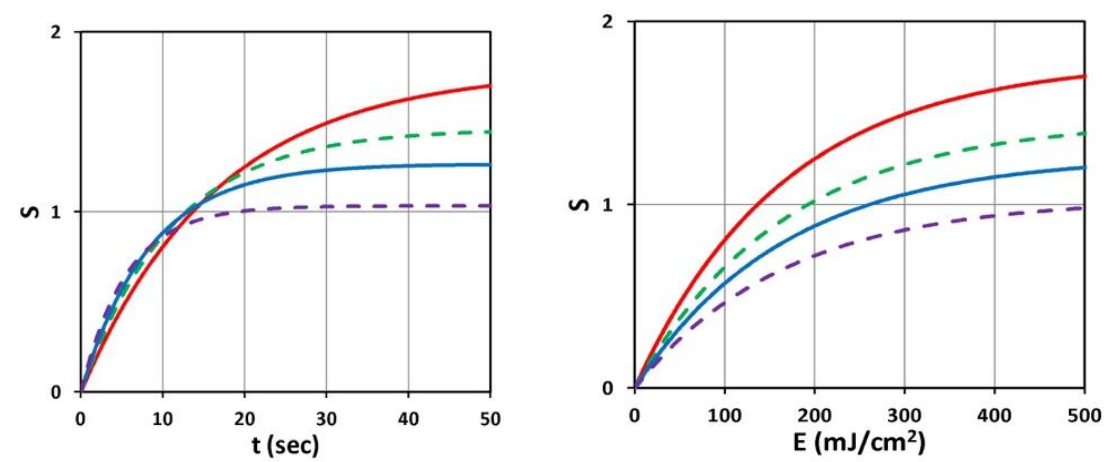

Fig. 4. Time profiles of $S$ versus $t$ (left figure) and versus dose $E_{0}$ (right figure) for various light intensity $\mathrm{I}_{0}=(10,15,20,30) \mathrm{mW} / \mathrm{cm}^{2}$, for $\mathrm{D}=1.0 \mathrm{~cm}, \mathrm{C}_{0}=3 \mathrm{mM}$, at $\mathrm{z}=0.5$ $\mathrm{cm}$.
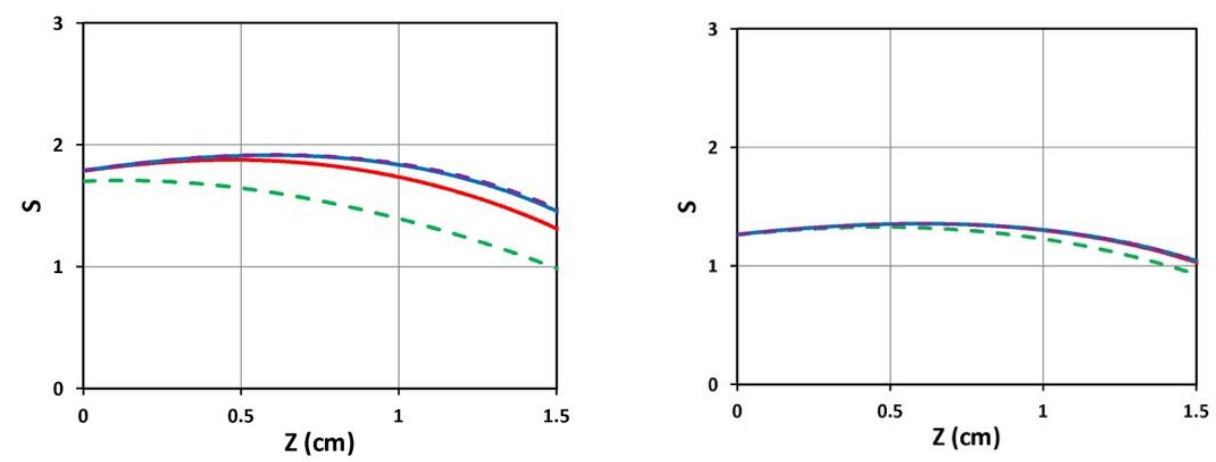

Fig. 5. Spatial profiles of $S$ for various exposure time $t=(100,200,300,400)$ (for curves low to top), for light intensity $\mathrm{I}_{0}=10$ (left figure) and 20 (right figure) $\mathrm{mW} / \mathrm{cm}^{2}$, and $\mathrm{D}=$ $1.0 \mathrm{~cm}, \mathrm{C}_{0}=2 \mathrm{mM}$ 

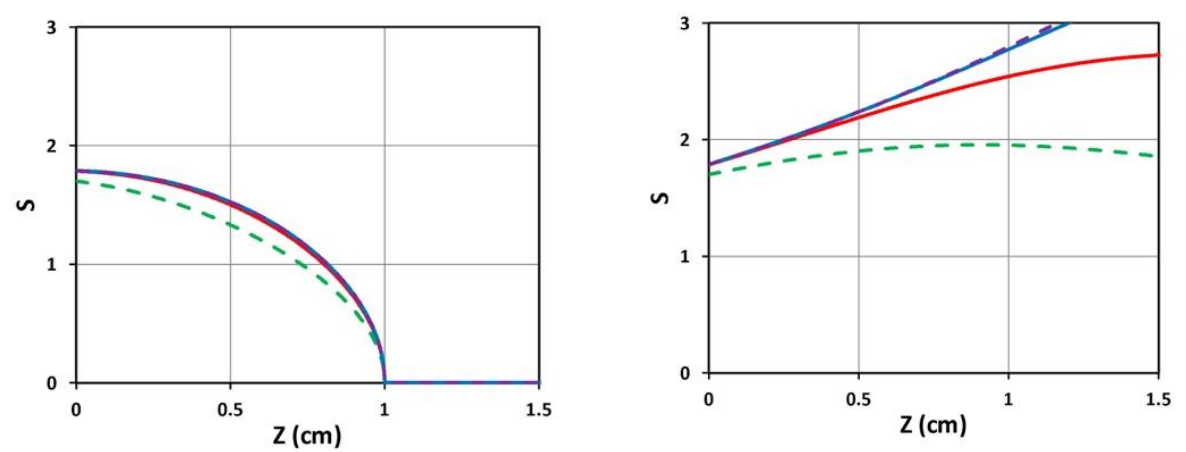

Fig. 6. Same as Fig. 5, but for $\mathrm{D}=0.5 \mathrm{~cm}$ (left figure) and $\mathrm{D}=20 \mathrm{~cm}$ (right figure), for light intensity $\mathrm{I}_{0}=10 \mathrm{~mW} / \mathrm{cm}^{2}$.
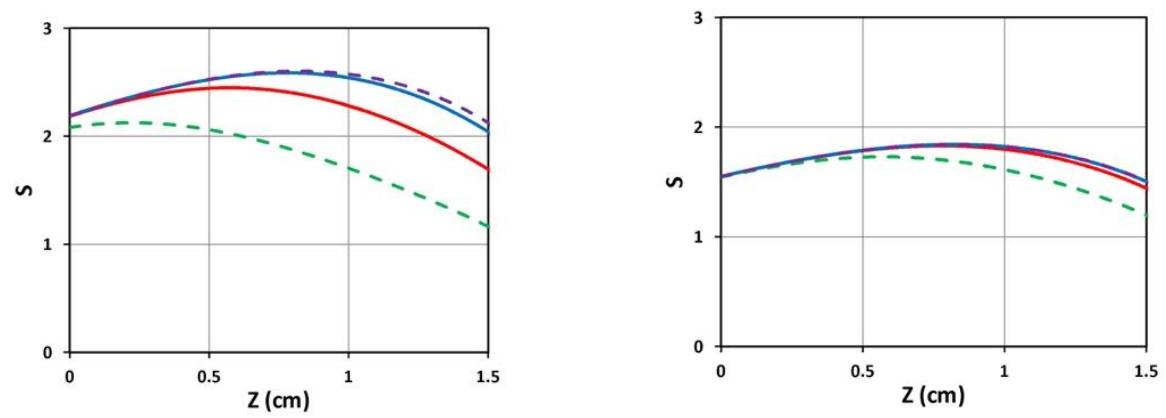

Fig. 7. Same as Fig. 5, but for higher $\mathrm{C}_{0}=3.0 \mathrm{mM}$.
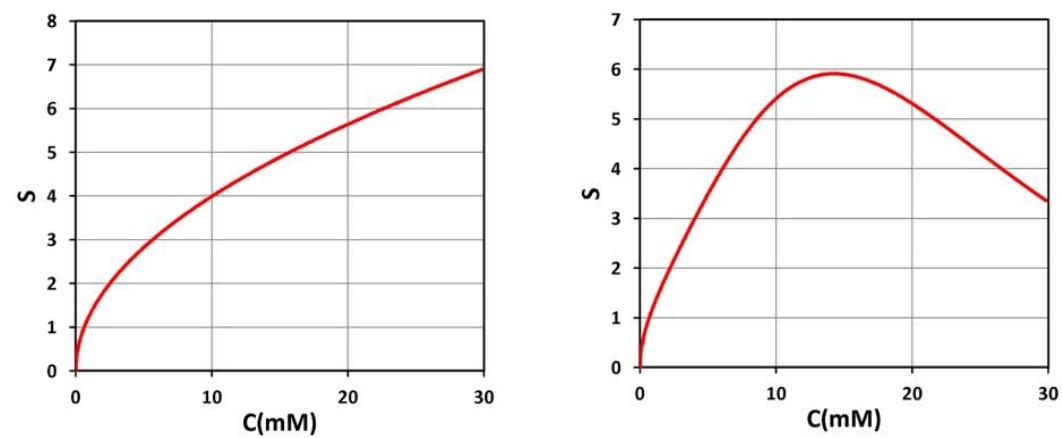

Fig. 8 shows $\mathrm{S}$ versus PS concentration $\left(\mathrm{C}_{0}\right)$, for $\mathrm{z}=0$ (left figure) and $\mathrm{z}=0.5 \mathrm{~cm}$ (right figure), for $\mathrm{D}=1.0 \mathrm{~cm}, \mathrm{I}_{0}=10 \mathrm{~mW} / \mathrm{cm}^{2}$.

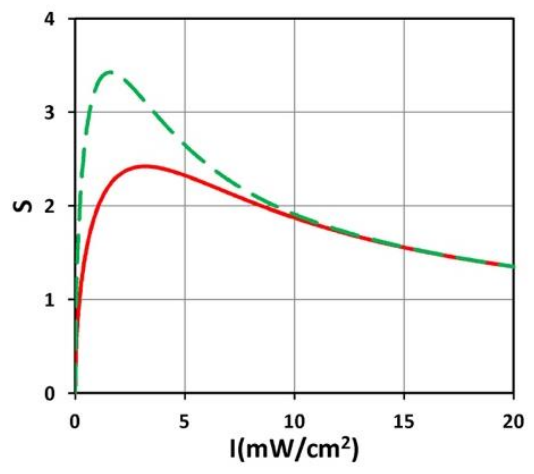


Fig. 9 shows $\mathrm{S}$ versus light intensity ( $\left.\mathrm{I}_{0}\right)$, for $\mathrm{t}=100 \mathrm{~s}$ (red curve) and $200 \mathrm{~s}$ (green curve), and

$\mathrm{z}=0.5 \mathrm{~cm}, \mathrm{D}=1.0 \mathrm{~cm}, \mathrm{C}_{0}=2 \mathrm{mM}$,

From the above figures, we are able to summarize the important features as follows.

(a) Fig. 4 (left figure) demonstrates that higher light intensity has a faster rising efficacy, but a lower steady-state value due to its faster depletion of PS concentration. As also shown by our formula, Eq. (5), that the efficacy at transient state (for small dose) is proportional to $\mathrm{tI}_{0}{ }^{0.5}$. However, at steady-state (with $\mathrm{E} 1=1$ ), it is a nonlinear function of $\left[\mathrm{C}_{0} / \mathrm{I}_{0}\right]^{0.5}$ or $\left[\mathrm{t} / \mathrm{E}_{0}\right]^{0.5}$. This nonlinear scaling law predicts the clinical data more accurate than the linear theory of Bunsen Roscoe law (BRL) [16].

(b) Fig. 4 (right figure) demonstrates that for the same dose $\left(\mathrm{E}_{0}\right)$, all light intensity has the same crosslink time, as shown by Eq. (4.b), E1=1-exp(-Bt), with $\mathrm{Bt}=\mathrm{bg}=\mathrm{aqg}\left(\mathrm{E}_{0}\right)$ which depends only on $\mathrm{E}_{0}$, independent to $\mathrm{I}_{0}$.

(c) As shown by Fig. 5 that higher light intensity has smaller efficacy, reduced by a factor of 1.43 when the intensity is doubled (for the same dose).

(d) As shown by Fig. 6, small diffusion depth (with $\mathrm{D}=1.0 \mathrm{~cm}$ ) has a more uniform but lower S profile; whereas for large D (or flat, uniform PS concentration) has higher efficacy, but non-uniform profile.

(e) As shown by Fig. 7, higher PS concentration (with $\mathrm{C}_{0}=3.0 \mathrm{mM}$ ) has higher by more non-uniform profile, comparing to Fig. 5 (with $\mathrm{C}_{0}=2.0 \mathrm{mM}$ ). It also shows a peak $\mathrm{S}$ at depth $z^{*}$, predicted by our analytic formula.

(f) As shown by Fig. 8, optimal PS concentration (for maximum S) exits for $\mathrm{z}>0$, but not for $\mathrm{z}=0$.

(g) Similarly, as shown by Fig. 9, there is an optimal light intensity (for a given time), but not for light dose (shown by Fig. 4). Moreover, as predicted by Eq. (4), higher intensity has smaller efficacy (at steady-state, E1=1).

\subsection{Strategies for optimal efficacy}

Base on our $\mathrm{z}^{*}$-formula, S-formula, Eq. (5), and S profiles shown by Figs. 5 to 9, we propose a new criterion of PPS efficacy based on the product of [strength] (or S1) and [depth] (or $z^{*}$ ), i.e., the [volume] of polymer being cross-linked. Furthermore, a threshold dose is required and this threshold limits the maximum light intensity (and a minimum crosslink time) as discussed earlier. For a given $\mathrm{C}_{0}$, deeper crosslink may be achieved by larger fluence $\left(\mathrm{E}_{0}\right)$. However, to achieve uniform and sufficient efficacy by a minimal $\mathrm{E}_{0}$, one requires an optimal range of $\mathrm{D}, \mathrm{I}_{0}$ and $\mathrm{C}_{0}$. An optimal goal is to gain 
maximum crosslink "volume", or [strength]x[depth], as well as polymerization uniformity, specially for thick polymer of 1.0 to $1.5 \mathrm{~cm}$.

We will discuss two classes of PPS: (a) synthetic polymer sample is prepared in a hydrogel form, where the PS solution is mixed in the gel uniformly; (b) nature polymer tissue (such as human cornea of skin), where the PS solution is diffused from its surface into the volume, having a controllable diffusion depth D.

Class (a) is related to our system with a fixed $F(z)=1$, and the initial PS concentration distribution (PSCD) can not be controlled by diffusion depth (D), for example, in photoinitiated polymerization of PEG-diacrylate hydrogel [4]. As shown by Fig. 6 (right figure), the gelation spatial profile (GSP), for the case of initially uniform PSCD (with $\mathrm{D}>>1.0 \mathrm{~cm}$, or F=1.0), is an increasing function of the depth (z), i.e., the anterior portion always has less efficacy (gelation). Strategy using two-side illumination [6,8] and focused light [7] were proposed to improve the overall efficacy and uniformity of the GSP.

For class (b), the PSCD profile is controlled by the diffusion time (or the value of D). As shown by Fig. 6 (left figure) with a non-uniform PSCD (with $\mathrm{D}=0.5 \mathrm{~cm}$ ), or Fig. 7 (with $\mathrm{D}=1.0 \mathrm{~cm}$ ) has a much more uniform crosslinked profile than that of uniform PSCD, except that the center portion (about 0.5 to $1.2 \mathrm{~cm}$ ) having slightly high efficacy than both ends (near the surface and the bottom). Comparing Fig. 5 (with $\mathrm{C}_{0}=2.0 \mathrm{mM}$ ) and Fig. 7 (with $\mathrm{C}_{0}=3.0 \mathrm{mM}$ ), we found that higher $\mathrm{C}_{0}$ has a worse crosslinked profile uniformity. However, low $\mathrm{C}_{0}$ having more uniformed PSCD, also results a low efficacy (comparing Fig. 5 and 7). Therefore, an optimal $\mathrm{C}_{0}$ should range between 1.5 and $3.0 \mathrm{mM}$.

Faster photoinitiated polymerization (gelation or crosslinking) maybe achieved by using a high intensity, which however, also results a low efficacy as shown in Fig. 5. To overcome the drawback of low efficacy in accelerated process using a high intensity, as predicted by our S-formula, a PS concentration-controlled method (CCM) was proposed recently by Lin in corneal crosslinking (CXL) [16]. Greater details based on the crosslink time ( $\left.\mathrm{T}^{*}\right)$ and the S-formula, Eq. (4) are discussed as follows.

As shown in Fig. 4 that higher light intensity has a faster rising efficacy, but a lower steady-state value due to its faster depletion of PS concentration. Therefore, resupply of PS drops, (with a supplying frequency defined as Fdrop), during the crosslink would improve the overall efficacy by a combined efficacy given by c-Eff= $1-\exp [-(\mathrm{S} 1+\mathrm{S} 2+\mathrm{S} 3+.)$.$] , where \mathrm{Sj}$ is the individual efficacy for each of the supply of PS. The time to re-supply PS is given by the the crosslink time defined earlier as $\mathrm{T}_{0}$ $\exp (\mathrm{Az})$, with the surface crosslink time given by $\mathrm{T}_{0}=4 /\left(\operatorname{aqg} \mathrm{I}_{0}\right)$, which is inverse proportional to the light intensity, absorption coefficient (a), quantum yield (q), and 
the effective kinetic rate constant (g) for type-I pathway.

We note that the Fdrop is proposed by the combined consideration of the crosslink time $\left(\mathrm{T}^{*}\right)$, which defines when PS is highly depleted (or time needed to reach the steady-state of efficacy); and the surface S-value, which defines the [strength] of crosslink, or the number of re-applying PS drops needed to achieve the same S-value for all intensity ranges (5 to $\left.100 \mathrm{~mW} / \mathrm{cm}^{2}\right)$. In Lin's proposed CCM [16], it predicts the comparable efficacy (for the same dose) for intensity of 1.5 to $45 \mathrm{~mW} / \mathrm{cm}^{2}$, based on a combined efficacy formula defined as c-Ceff=1-exp [$(\mathrm{S} 1+\mathrm{S} 2+. . \mathrm{Sj})]$, with $\mathrm{j}=\mathrm{Fdrop}$, and Fdrop is given by the integer-portion of squareroot of $\left[\mathrm{I}_{0} / 3\right] \mathrm{xN}$, with $\mathrm{N}=$ Fdrop, being the Fdrop for optimal efficacy at the referenced intensity $\left(3 \mathrm{~mW} / \mathrm{cm}^{2}\right)$ in corneal crosslink (CXL). For other PPS, if the referenced intensity is $10 \mathrm{~mW} / \mathrm{cm}^{2}$, then Fdrop $=3$ or 4 , is required to achieve the similar efficacy when for a higher intensity of $100 \mathrm{~mW} / \mathrm{cm}^{2}$. Our S-formula shows that the steadystate $\mathrm{S}$ for $\mathrm{I}_{0}=10 \mathrm{~mW} / \mathrm{cm}^{2}$ is 1.43 (or squre-root of 2) lower than that of $\mathrm{I}_{0}=10$ $\mathrm{mW} / \mathrm{cm}^{2}$, when no re-supply of PS is administered (or Fdrop=1). Fig. 10 shows a schematic example of the combined S-function with Fdrop=3, with first PS instillation having $\mathrm{D}_{1}=1.0 \mathrm{~cm}$, followed by $\mathrm{D}_{2}=0.5 \mathrm{~cm}$ and $\mathrm{D}_{3}=0.2 \mathrm{~cm}$. It shows that the combined efficacy of high intensity, $\mathrm{I}_{0}=100 \mathrm{~mW} / \mathrm{cm}^{2}$, (curve-3, with Fdrop=3) achieves a similar efficacy as that of low intensity, $\mathrm{I}_{0}=10 \mathrm{~mW} / \mathrm{cm}^{2}$, (with Fdrop=0). And the total illumination time ( $\mathrm{t}$ ) for high intensity at $\mathrm{I}_{0}=100 \mathrm{~mW} / \mathrm{cm}^{2}$, is approximately $\mathrm{t}=3\left(\mathrm{~T}_{1} / 10\right)=0.3 \mathrm{~T}_{1}$, which is 3.3 times shorter than $\mathrm{T}_{1}$, the crosslink time for $\mathrm{I}_{0}=10 \mathrm{~mW} / \mathrm{cm}^{2}$. If one includes the waiting time for enough PS diffusion during each of the re-supply PS (approximately $0.2 \mathrm{~T}_{1}$ ), the total illumination time for high intensity is still 2 times faster.

Above is an example of strategy for optimal protocol for accelerated procedure (using a high intensity) without reducing its efficacy. While our theoretical predictions provide quantitative guidance or strategy for optimal PPS efficacy, their accuracy depends on accurate measured parameters of absorption coefficient (a), quantum yield (q), and the effective kinetic rate constant (g) for type-I pathway, besides further laboratory studies. 


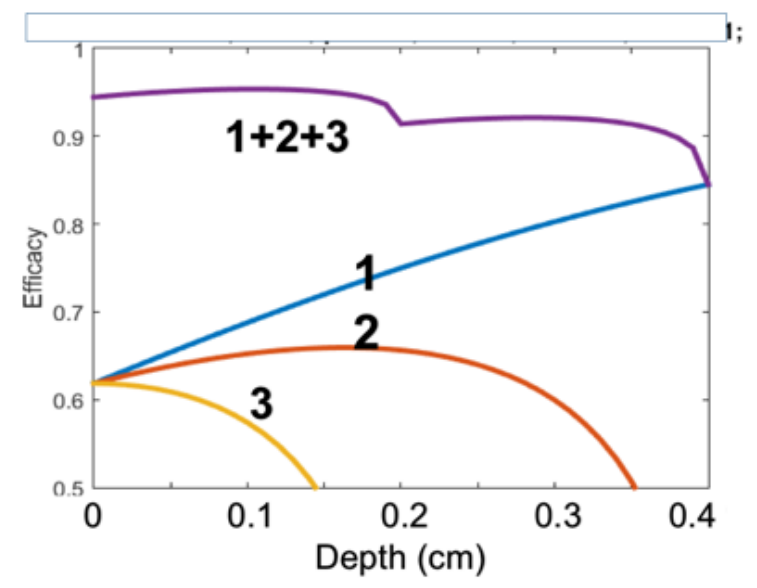

Fig. 10 The combined S-function, for Fdrop=3, where top curve is the combined function of curve1, 2, and 3 showing a high efficacy than curve 1 (with Fdrop=0).

\section{Conclusion}

The overall PPS efficacy is proportional to the UV light dose (or fluence), the PS initial concentration and their diffusion depths. An optimal goal is to gain fast and maximum crosslink "volume", or [strength]x[depth], as well as polymerization uniformity. A new proposed strategy using concentration-controlled method can improve the efficacy in accelerated PPS, which is less efficient than the low intensity (with the same dose) under the normal, non-controlled method.

\section{Acknowledgments}

This work was supported by the internal grant of New Vision Inc. KT Chen is partially supported by the Ph.D program of Graduate Institute of Applied Science and Engineering, Fu Jen Catholic University, Taiwan. HW Liu is supported by the National Science Council, NSC 1XXXX .

\section{Author Contributions}

Conceptualization, Jui-Teng Lin; Data curation, Jui-Teng Lin and Da-Chuan Cheng; Formal analysis, Jui-Teng Lin; Funding acquisition, Hsia-Wei Liu and Da-Chuan Cheng; Methodology, Jui-Teng Lin; Project administration, Hsia-Wei Liu; Software, Kuo-Ti Chen and Da-Chuan Cheng; Supervision, Jui-Teng Lin.

\section{Conflicts of Interest}


Jui-Teng Lin is the CEO of New Vision Inc.

\section{REFERENCES}

1. Fouassier J-P. Photoinitiation, Photo-polymerization, and Photocuring: Fundamentals and Applications. 1995, Hanser Gardner Publications Munich.

2. Odian G. Principles of Polymerization. 1991, Wiley, New York.

3. Bryant SJ, Nuttelman CR, Anseth KS (2000) Cytocompatibility of UV and visible light photoinitiating systems on cultured NIH/3T3 fibroblasts in vitro J Biomater Sci Poly 11:439-457

4. Fairbanks BD, Schwartz MP, Bowman CN, Anseth KS. Photoinitiated polymerization of PEG-diacrylate with lithium phenyl-2,4,6trimethylbenzoylphosphinate: Polymerization rate and cytocompatibility. Biomaterials. 2009, 30:6702-6707

5. Terrones G, Pearlstein AJ. Effects of optical attenuation and consumption of a photobleaching initiator on local initiation rates in photopolymerizations. Macromolecules. 2001, 34: 3195-3204

6. Kenning NS, Kriks D, El-Maazawi M, Scranton A. Spatial and temporal evolution of the photo initiation rate for thick polymer systems illuminated on both sides. Polym Int. 2005, 54: 1429-1439

7. Lin JT, Liu HW. Cheng DC. Optimal focusing and scaling law for uniform photo-polymerization in a thick medium using a focused UV Laser. Polymers. 2014, 6:552-564, i:10.3390/polym6020552.

8. Lin JT, Liu HW. Cheng DC. Modeling the kinetics of enhanced photopolymerization under a collimated and a reflecting focused UV laser. Polymers. 2014, 6: 1489-1501.

9. Lin JT, KC Wang. Analytic formulas for the dynamics of non-uniform polymerization by a UV light. J Polym Res. 2016, 23: 53 DOI 10.1007/s10965016-0934-4.

10. Lin JT, Chen KT, Liu HW. Novel techniques for improving anti-cancer efficacy via synergistic phototherapy. Op Acc J Bio Eng \& App. 291)-2018. OAJBEA.MS.ID.000126.

11. Lin JT, Chen KT, Liu HW. Analysis of kinetics and efficacy of anti-cancer via oxygen-enhanced photodynamic therapy. J Cancer Research update, 2018:7:2126.

12. Zhu TC, Finlay JC, Zhou X, et al. Macroscopic Modeling of the singlet oxygen production during PDT. Proc SPIE. 2007; 6427:6427081-64270812.

13. Kim MM, Ghogare AA, Greer A, Zhu TC et al. On the in vivo photochemical rate parameters for PDT reactive oxygen species modeling. Phys. Med. Biol. 62 (2017) R1-R48. 
14. Lin JT, Cheng DC. Modeling the efficacy profiles of UV-light activated corneal collagen crosslinking. PloS One. 2017;12:e175002. DOI:10.1371/journal.pone.0175002.

15. Lin JT. A proposed concentration-controlled new protocol for optimal corneal crosslinking efficacy in the anterior stroma. Invest. Ophthalmol Vis Sci. 2018;59:431-432.

16. Lin JT. Efficacy S-formula and kinetics of oxygen-mediated (type-II) and nonoxygen-mediated (type-I) corneal cross-linking. Ophthalmology Research. 2018; 8(1): 1-11, Article no.OR.39089. DOI : 10.9734/OR/2018/39089.

17. Lin JT, Liu HW, Cheng DC. On the dynamic of UV-Light initiated corneal crosslinking. J. Med Biolog Eng. 2014;34:247-250; doi: 10.5405/jmbe.15332. 